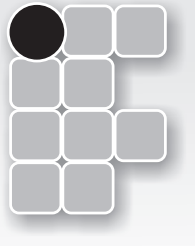

\title{
MONITORAMENTO DA QUALIDADE DAS ÁGUAS DO RIO DO OURO, EM OURO VERDE DO OESTE - PR: ANÁLISES TOXICOLÓGICAS
}

Daniele Cristina Schons

Daniel Schwantes ${ }^{2}$

Valdemir Aleixo ${ }^{3}$

Nayara Szymanski ${ }^{4}$

Michelli Caroline Ferronato ${ }^{5}$

Affonso Celso Gonçalves Junior ${ }^{6}$

Victor Emidio Pertile

Lucas Beckhauser de Moraes $^{8}$

\section{Resumo}

A preocupação mundial com a conservação dos recursos hídricos é causa de conflitos, sendo a escassez de água potável provocada pelas mudanças climáticas e principalmente por ações antrópicas. O objeto de estudo deste projeto é o Rio do Ouro, localizado na cidade de Ouro Verde do Oeste, distante $560 \mathrm{~km}$ da capital do estado, Curitiba. Os pontos de coleta são acompanhados pela urbanização com áreas de atividades agrícolas, suinocultura e piscicultura. Localizado entre os municípios de Toledo e Ouro Verde do Oeste no estado do Paraná, sua extensão em linha reta é de $22 \mathrm{~km}$. 0 objetivo do presente trabalho é avaliar a qualidade da água do Rio do Ouro por meio de avaliação das condições toxicológicas. Este estudo trará contribuições importantes para a elaboração e implantação de práticas de conservação e gestão ambiental, além de servir como base de dados para estudos posteriores sobre o Rio do Ouro. Os teores médios dos metais obtidos durante o período de amostragem indicam baixo impacto ambiental, considerando as atividades agroindustriais e atividade urbana presentes, porém, cabe salientar que estas atividades poderão causar maiores impactos ao referido curso hídrico futuramente, caso o manejo conservacionista das áreas marginais seja abandonado.

Palavras-chave: Recursos hídricos. Avaliação toxicológica. Poluição. Paraná.

\section{Introdução}

Cerca de $97,5 \%$ da água de nosso planeta está presente nos oceanos e mares, na forma de água salgada, ou seja, imprópria para o consumo humano. Dos 2,5\% restantes, que perfazem o total de água doce existente, 2/3 estão armazenados nas geleiras e calotas polares. Apenas aproximadamente 0,77\% de toda a água está disponível para o nosso consumo, sendo encontrada na forma de rios, lagos, água subterrânea, incluindo ainda a água presente no solo, atmosfera (umidade) e na biota.

${ }^{1}$ Graduanda do Curso de Engenharia Ambiental; Pontifícia Universidade Católica do Paraná; Toledo - PR.

E-mail: daniele.schons@outlook.com.

${ }^{2}$ Professor orientador; Escola Politécnica; Pontifícia Universidade Católica do Paraná; Marechal Cândido Rondon - PR.

E-mail: daniel.schwantes@pucpr.br.

${ }^{3}$ Professor orientador; Coordenador do Curso de Engenharia Ambiental; Escola politécnica; Pontifícia Universidade Católica do Paraná;

Toledo - PR. E-mail: valdemir.aleixo@pucpr.br.

${ }^{4}$ Professor orientador; Escola Politécnica; Pontifícia Universidade Católica do Paraná; Toledo - PR. E-mail: nayara.szymanski@pucpr.br.

${ }^{5}$ Professor orientador; Escola Politécnica; Pontifícia Universidade Católica do Paraná; Toledo - Paraná. E-mail: michelli.ferronato@pucpr.br.

${ }^{6}$ Professor; Universidade Estadual do Oeste do Paraná; Marechal Cândido Rondon - PR. E-mail: affonso133@hotmail.com.

${ }^{7}$ Graduando do Curso de Engenharia Ambiental; Pontifícia Universidade Católica do Paraná; Cascavel - PR.

E-mail: victor.pertile@outlook.com.

${ }^{8}$ Graduando do Curso de Engenharia Ambiental; Pontifícia Universidade Católica do Paraná; Toledo - PR.

E-mail: lucasbeck7@hotmail.com. 
O Brasil está dividido em doze regiões hidrográficas, dentre elas a do Paraná, a qual, em termos de águas superficiais detém $6,4 \%$ dos recursos hídricos e é considerada a terceira maior região hidrográfica do país (BRASIL, 2014a). Esta região engloba os estados de São Paulo, Paraná, Mato Grosso do Sul, Minas Gerais, Goiás, Santa Catarina e o Distrito Federal (BRASIL, 2014b).

A hidrografia do estado do Paraná está dividida em dezesseis bacias hidrográficas, dentre as quais se encontra a Bacia do Paraná 3, onde se localiza o objeto do presente estudo, o Rio do Ouro (PARANÁ, 2010). Está localizado entre os municípios de Toledo e Ouro Verde do Oeste, no Paraná. Possui extensão em linha reta de 22 km, conforme dados da Prefeitura Municipal de Ouro Verde do Oeste - PR. Os pontos de coleta estão localizados em sua totalidade no município de Ouro Verde do Oeste, que possui 5.927 habitantes e está distante $560 \mathrm{~km}$ da capital do estado, Curitiba (IBGE, 2013).

A nascente do Rio do Ouro encontra-se numa área de mata fechada. Porém os demais trechos possuem em seu entorno atividades agrícolas, suinocultura, piscicultura e urbanização; além de possuírem a ocupação urbana muito próxima do leito do rio, não respeitando o limite mínimo de proteção de matas ciliares, que é de 30 metros, de acordo a Lei n. ${ }^{\circ} 12.651$, de 25 de maio de 2012 (BRASIL, 2012).

Segundo Philippi Jr. (2005), em um ecossistema rural, o conjunto de atividades agropecuárias pode ser responsável por mudanças significativas no meio primitivo. No caso de estudo, alterações na mata ciliar poderiam estar resultando em impacto ambiental ao rio por meio da erosão e lixiviação dos solos agrícolas marginais ao rio.

Além disso, a ocupação urbana também causa prejuízos à qualidade da água. Segundo a Secretaria de Estado do Meio Ambiente e Recursos Hídricos (PARANÁ, 2013, p. 7) "todas as atividades realizadas na bacia desenvolvidas por indústrias, propriedades rurais e cidades refletem na qualidade da água do rio, desde suas nascentes até a sua foz".

Conforme a Prefeitura Municipal de Ouro Verde do Oeste e observações feitas em campo, não existe o lançamento direto de esgoto doméstico no rio, somente águas pluviais, porém, avaliações microbiológicas realizadas por Schons et al. (2014), paralelamente a este estudo no Rio do Ouro evidenciaram a possibilidade de contaminação por fezes. Além disso, sabe-se que o lixo, quando depositado inadequadamente nas ruas, pode acompanhar o fluxo da água e na ocorrência de altas precipitações pluviométricas, ser despejado no rio juntamente com as águas pluviais, ocasionando a contaminação do corpo hídrico.

Os impactos sobre os recursos hídricos são preocupantes, não só no aspecto quantitativo, mas também no qualitativo. Além disso, na área rural, para viabilizar a produção, são utilizados produtos químicos como fertilizantes e agrotóxicos, fazendo recair sobre os produtores a responsabilidade pela contaminação do meio e perdas ambientais.

O presente trabalho objetivou avaliar as concentrações de metais e de alguns nutrientes presentes nas águas do Rio do Ouro, em Ouro Verde do Oeste, visando caracterizar os possíveis impactos ambientais ocasionados pela ação antrópica na região.

\section{Material e Métodos}

O monitoramento do Rio do Ouro foi realizado durante o período de novembro de 2012 a novembro de 2013. Inicialmente foi realizada a seleção, o reconhecimento dos pontos de amostragem e a locação das coordenadas geográficas. Foi firmado, também um acordo com a prefeitura municipal, com finalidade de auxílio na realização das amostragens durante o período avaliado.

Na Tabela 01 e no Quadro 01 são apresentadas informações dos pontos de amostragem, suas coordenadas geográficas e atividades de entorno, na Figura 01 está ilustrada a localização dos pontos de coleta em relação ao Município de Ouro Verde do Oeste e na Figura 2 estão apresentadas imagens dos pontos de coleta. 
Tabela 01. Coordenadas dos pontos selecionados, Rio do Ouro, Ouro Verde do Oeste-PR.

\begin{tabular}{cc}
\hline PONTOS AMOSTRAIS & \multicolumn{1}{c}{ COORDENADAS } \\
\hline $\mathbf{2}$ & $24^{\circ} 48^{\prime} 06.09^{\prime \prime} \mathrm{S} / 53^{\circ} 52^{\prime} 12.68^{\prime \prime} \mathrm{O}$ \\
$\mathbf{3}$ & $24^{\circ} 47^{\prime} 35.47^{\prime \prime} \mathrm{S} / 53^{\circ} 53^{\prime} 07.59^{\prime \prime} \mathrm{O}$ \\
4A & $24^{\circ} 47^{\prime} 42.65^{\prime \prime} \mathrm{S} / 53^{\circ} 54^{\prime} 11.10^{\prime \prime} \mathrm{O}$ \\
4B & $24^{\circ} 47^{\prime} 39.17^{\prime \prime} \mathrm{S} / 53^{\circ} 54^{\prime} 06.84^{\prime \prime} \mathrm{O}$ \\
$\mathbf{5}$ & $24^{\circ} 48^{\prime} 00.95^{\prime \prime} \mathrm{S} / 53^{\circ} 54^{\prime} 54.30^{\prime \prime} \mathrm{O}$ \\
\hline
\end{tabular}

Fonte: Prefeitura Municipal de Ouro Verde do Oeste - PR, 2013.

Quadro 01. Incidência de atividade agroindustrial nas imediações dos pontos de amostragem, Rio do Ouro, Ouro Verde do Oeste-PR.

\begin{tabular}{ccccc}
\hline PONTOS & AGRICULTURA & AVICULTURA & PISCICULTURA & URBANIZAÇÃO \\
\hline 2 & $\mathrm{X}$ & & $\mathrm{X}$ & \\
3 & $\mathrm{X}$ & $\mathrm{X}$ & $\mathrm{X}$ & \\
4A & $\mathrm{X}$ & & & $\mathrm{X}$ \\
4B & $\mathrm{X}$ & & & \\
5 & $\mathrm{X}$ & & & \\
\hline
\end{tabular}

Fonte: Prefeitura Municipal de Ouro Verde do Oeste - PR, 2013.

Nota: X Refere-se à presença da atividade nas imediações dos pontos de amostragem.

As amostragens foram realizadas mensalmente durante o período de novembro de 2012 a novembro de 2013. As datas estipuladas para as amostragens foram respeitadas, sendo a frequência de amostragens de aproximadamente quinze dias.

A prefeitura Municipal de Ouro Verde do Oeste disponibilizou botas, luvas e outros materiais para coleta. A Pontifícia Universidade Católica do Paraná - Campus Toledo disponibilizou seus laboratórios, freezer para acondicionamento após coleta, caixas térmicas, fitas, canetas e papel filme. A Universidade Estadual do Paraná em Marechal Cândido Rondon disponibilizou o laboratório de Química Ambiental e Instrumental para realização das análises toxicológicas.

As concentrações dos metais: potássio $(\mathrm{K})$, cálcio $(\mathrm{Ca})$, magnésio $(\mathrm{Mg})$, cobre $(\mathrm{Cu})$, zinco $(\mathrm{Zn})$, ferro (Fe), manganês $(\mathrm{Mn})$, cádmio $(\mathrm{Cd})$, chumbo $(\mathrm{Pb})$ e cromo $(\mathrm{Cr})$ foram determinadas por espectrometria de absorção atômica modalidade chama (EAAlchama) (Welz; Sperling, 1999).

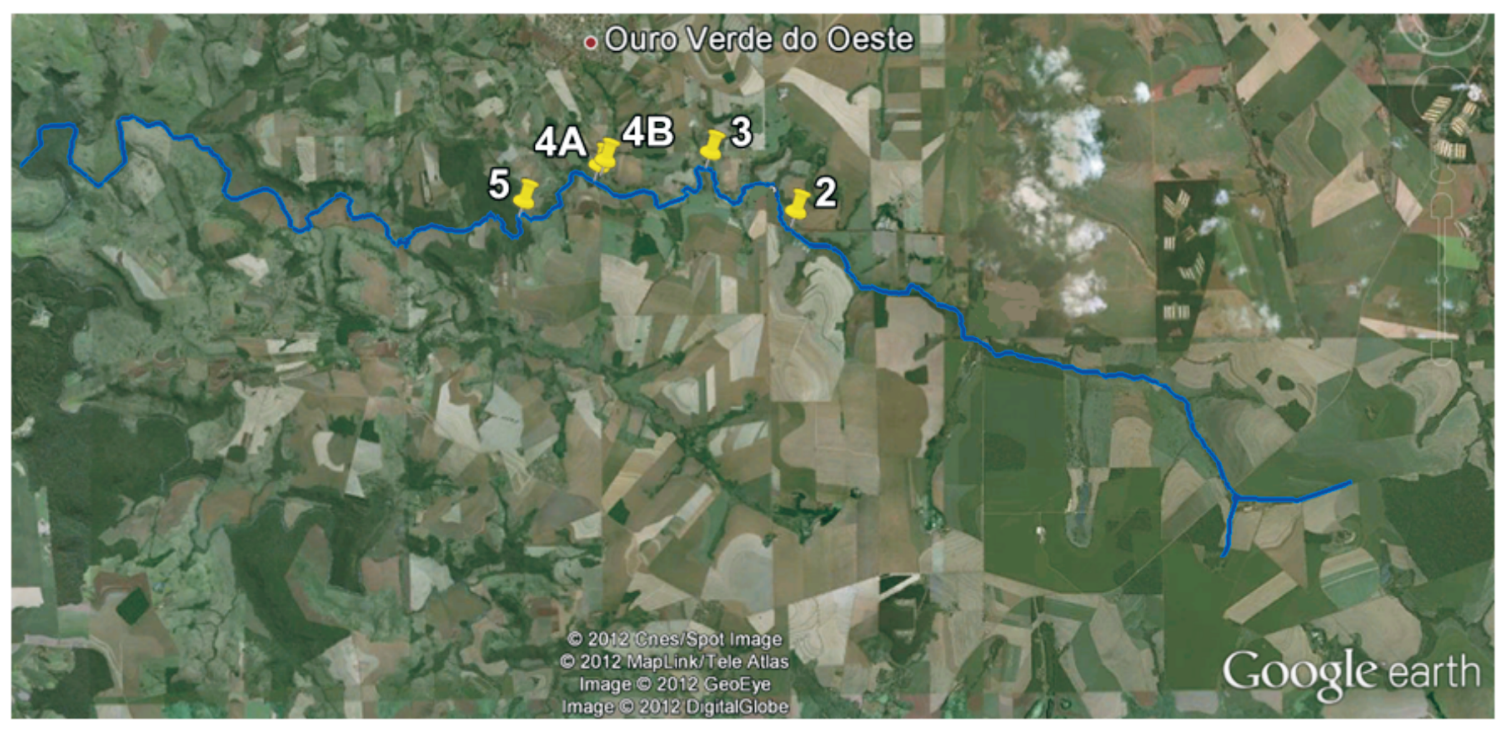

Figura 01. Localização dos pontos amostrais georreferenciados.

Fonte: Prefeitura Municipal de Ouro Verde do Oeste - PR, 2013. 


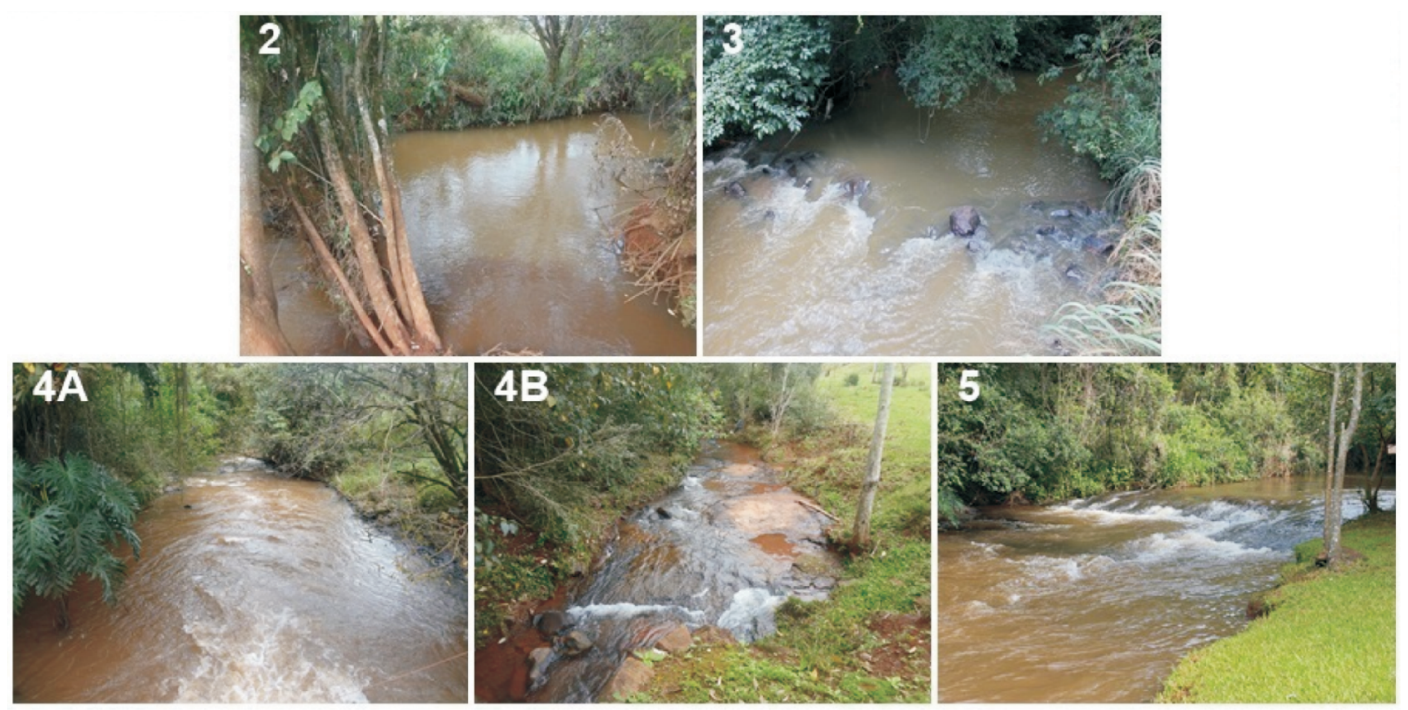

Figura 02. Pontos de coleta definidos para análises no Rio do Ouro.

Fonte: Elaboração dos autores, 2014.

Os valores médios dos teores dos elementos analisados durante o período de amostragem foram comparados com os valores máximos da Resolução n. ${ }^{\circ} 357$ do Conselho Nacional do Meio Ambiente (CONAMA), de 17 de março de 2005 (BRASIL, 2005b), para águas doces de classe 3 — classe em que o rio se enquadra - e, quando inexistente o padrão na resolução anterior, o valor médio foi comparado com a Resolução da Diretoria Colegiada da Agência Nacional de Vigilância Sanitária (Anvisa) n. ${ }^{\circ} 274$ de 22 de setembro de 2005 (BRASIL, 2005a) para águas adicionadas de sais destinadas a consumo humano.

Foi utilizado o programa OriginPro 8.0 para a apresentação gráfica dos resultados obtidos.

\section{Resultados e Discussão}

Durante o período amostral foram monitorados alguns parâmetros meteorológicos, tais como umidade relativa (UR \%), temperatura máxima, mínima, média e precipitação pluviométrica média (Figura 02).

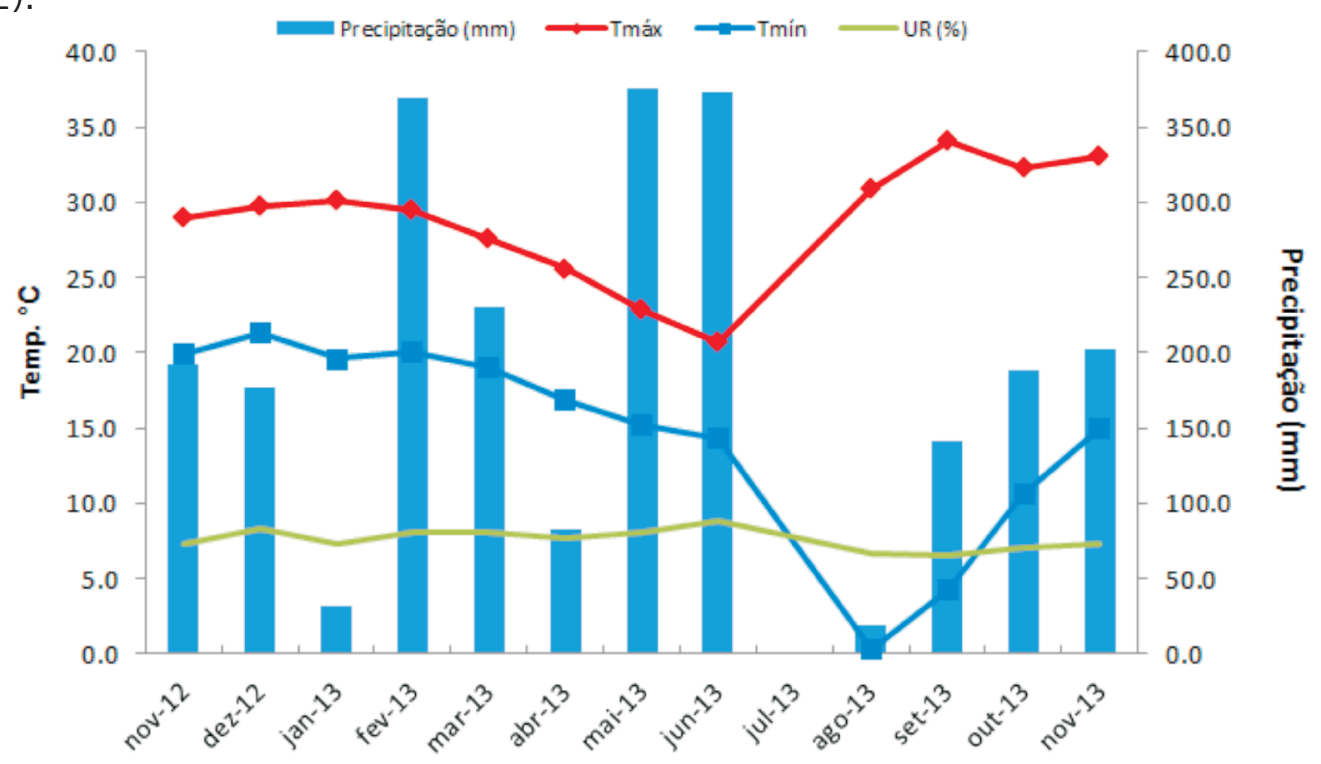

Figura 02. Dados climatológicos monitorados no período de novembro/2012 a novembro/2013. Fonte: Dados da estação climatológica da PUCPR, Campus Toledo. 
A comparação entre os valores médios obtidos e a legislação estão apresentados na Tabela 02. Na Tabela 03 são apresentados os valores médios dos teores dos elementos em função dos pontos de amostragem, durante todo o período de monitoramento.

Tabela 02. Resultados médios obtidos durante o período de novembro de 2012 a novembro de 2013 em comparação com os valores máximos permitidos pela Resolução do CONAMA n. ${ }^{\circ}$ 357/05 para águas doces de Classe 3 e com os valores máximos permitidos pela Resolução da Anvisa n. ${ }^{\circ}$ 274/05 para águas adicionadas de sais destinadas a consumo humano.

\begin{tabular}{ccccccccccc}
\hline Parâmetros & $\mathbf{K}$ & $\mathbf{C a}$ & $\mathbf{M g}$ & $\mathbf{C u}$ & $\mathbf{Z n}$ & $\mathbf{M n}$ & $\mathbf{F e}$ & $\mathbf{C d}$ & $\mathbf{P b}$ & $\mathbf{C r}$ \\
\hline $\begin{array}{c}\text { Unidade } \\
\text { Valor máximo }\end{array}$ & $500,0^{\mathrm{a}}$ & $250,0^{\mathrm{a}}$ & $65,0^{\mathrm{a}}$ & $0,013^{\mathrm{b}}$ & $5,000^{\mathrm{b}}$ & $0,500^{\mathrm{b}}$ & $5,000^{\mathrm{b}}$ & $0,010^{\mathrm{b}}$ & $0,033^{\mathrm{b}}$ & $0,050^{\mathrm{b}}$ \\
\hline $\begin{array}{c}\text { Média de 12 } \\
\text { meses }\end{array}$ & 0.942 & 1.494 & 1.284 & - & - & - & 0.084 & - & 0.018 & 0.034 \\
\hline
\end{tabular}

Fonte: BRASIL (2005a); BRASIL (2005b); Elaboração dos autores, 2014.

Nota: ${ }^{a}$ refere-se a padrões estabelecidos pela Anvisa; ${ }^{b}$ refere-se a padrões do CONAMA.

Observam-se, na Tabela 03, concentrações quanto aos teores de $\mathrm{Cu}, \mathrm{Zn}, \mathrm{Mn}$ e Cd inferiores ao limite de quantificação, o que sugere que estes metais estejam presentes em concentrações extremamente baixas.

Tabela 03. Valores médios dos teores dos elementos em função dos pontos de amostragem no Rio do Ouro em Ouro Verde do Oeste - PR entre novembro de 2012 e novembro de 2013.

\begin{tabular}{|c|c|c|c|c|c|c|c|c|c|c|c|}
\hline & & $\mathbf{K}$ & $\mathrm{Ca}$ & Mg & $\mathrm{Cu}$ & $\mathrm{Zn}$ & Mn & $\mathrm{Fe}$ & Cd & $\mathrm{Pb}$ & $\mathrm{Cr}$ \\
\hline & & \multicolumn{10}{|c|}{$\mathrm{mg} \mathrm{L}^{-1}$} \\
\hline \multirow{5}{*}{ 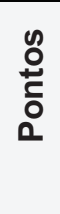 } & 2 & $0.78 \pm 0.50$ & $1.20 \pm 0.14$ & $1.04 \pm 0.43$ & $<0.005$ & $<0.005$ & $<0.01$ & $0.09 \pm 0.13$ & $<0.005$ & $0.01 \pm 0.02$ & $0.05 \pm 0.07$ \\
\hline & 3 & $0.81 \pm 0.53$ & $1.21 \pm 0.22$ & $1.12 \pm 0.44$ & $<0.005$ & $<0.005$ & $<0.01$ & $0.07 \pm 0.09$ & $<0.005$ & $0.01 \pm 0.03$ & $0.02 \pm 0.04$ \\
\hline & 4A & $1.11 \pm 0.89$ & $1.41 \pm 0.32$ & $1.29 \pm 0.27$ & $<0.005$ & $<0.005$ & $<0.01$ & $0.09 \pm 0.14$ & $<0.005$ & $0.02 \pm 0.06$ & $0.03 \pm 0.04$ \\
\hline & 4B & $1.06 \pm 0.38$ & $1.82 \pm 0.66$ & $1.55 \pm 0.24$ & $<0.005$ & $<0.005$ & $<0.01$ & $0.10 \pm 0.14$ & $<0.005$ & $0.03 \pm 0.10$ & $0.02 \pm 0.04$ \\
\hline & 5 & $0.95 \pm 0.46$ & $1.83 \pm 0.66$ & $1.42 \pm 0.20$ & $<0.005$ & $<0.005$ & $<0.01$ & $0.07 \pm 0.08$ & $<0.005$ & $0.02 \pm 0.07$ & $0.05 \pm 0.07$ \\
\hline
\end{tabular}

Fonte: Elaboração dos autores, 2014.

Nota: $n=9$. Limite de Quantificação: $\mathrm{Cu}=0.005 \mathrm{mg} \mathrm{L}^{-1} ; \mathrm{Zn}=0.005 \mathrm{mg} \mathrm{L}^{-1} ; \mathrm{Mn}=0.01 \mathrm{mg} \mathrm{L}^{-1} ; \mathrm{Cd}=0.005 \mathrm{mg}$ $\mathrm{L}^{-1}$. 
Na Figura 03 é apresentada a variação obtida para os teores de K, Ca e Mg durante o período de amostragem.

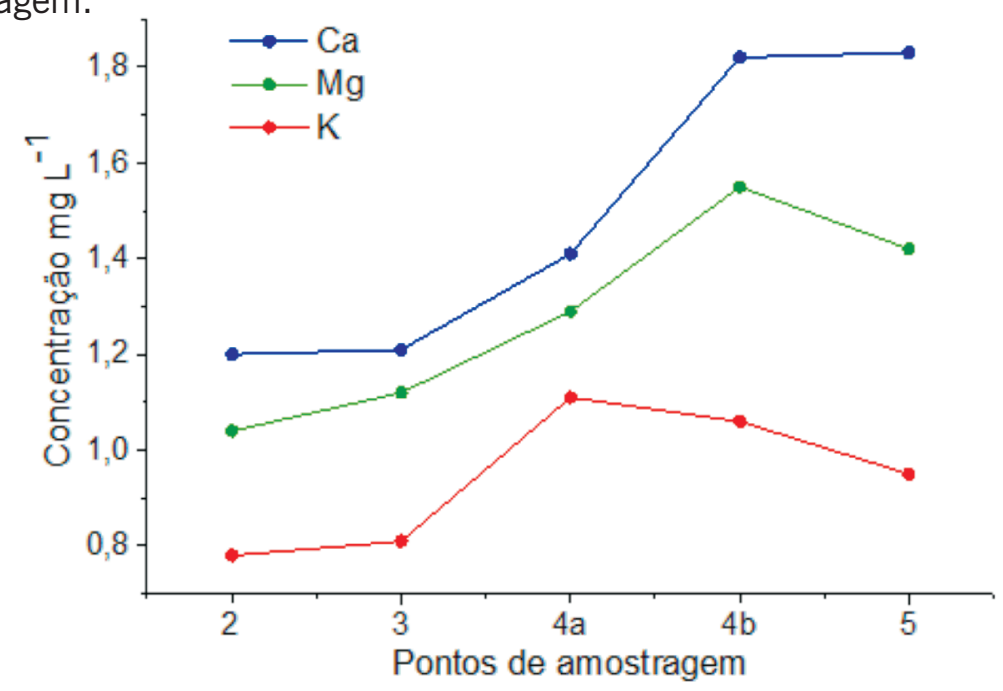

Figura 3. Valores médios das concentrações dos metais $\mathrm{K}$, Ca e Mg nos pontos de amostragem do Rio do Ouro, em Ouro Verde do Oeste - PR, entre novembro de 2012 e novembro de 2013.

Fonte: Elaboração dos autores, 2014.

Observam-se maiores médias quanto aos pontos de amostragem 4B e 5 para os metais Ca e Mg, enquanto que observam-se maiores concentrações de $\mathrm{K}$ nos pontos $4 \mathrm{~A}$ e $4 \mathrm{~B}$.

As concentrações de K, Ca e Mg não são consideradas pela maior parte das legislações vigentes como parâmetro para caracterização da água ou para lançamento de efluentes, porém, os teores desses metais são previstos pela Anvisa (BRASIL, 2005a) como parâmetros da qualidade da água para consumo humano. Observa-se que os valores médios obtidos para as concentrações de $\mathrm{K}, \mathrm{Ca}$ e $\mathrm{Mg}$ estão bem abaixo dos valores máximos permitidos por esta legislação (Tabela 02).

$\mathrm{Na}$ Figura 04 são apresentadas as concentrações dos metais $\mathrm{Pb}$ e $\mathrm{Cr}$ nos pontos de amostragem avaliados.

Observa-se na Figura 04 teores médios de $\mathrm{Cr}$ e $\mathrm{Pb}$ muito próximos ao limite máximo estabelecido pelo CONAMA em águas doces de classe 3 , sendo este um possível indicativo de atividades antrópicas que venham a contaminar os cursos hídricos com esses metais, como a suinocultura ou o lançamento de efluentes industriais que tenham tais metais em sua composição.

$\mathrm{Na}$ Tabela 04 são apresentados os teores médios dos elementos durante o período de amostragem.

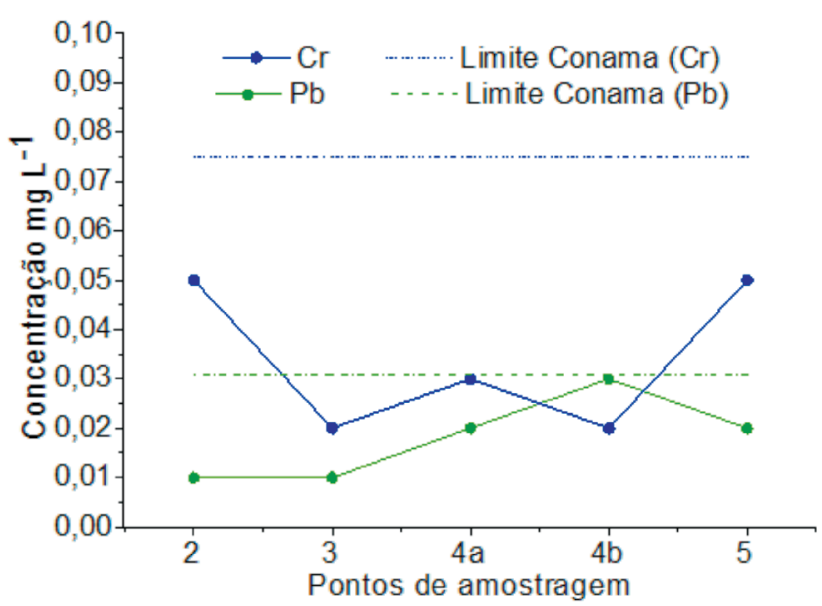

Figura 04. Valores médios das concentrações dos metais pesados $\mathrm{Pb}$ e $\mathrm{Cr}$ nos pontos amostrais do Rio do Ouro, em Ouro Verde do Oeste - PR, entre novembro de 2012 e novembro de 2013.

Fonte: Elaboração dos autores, 2014. 
Tabela 04. Valores médios dos teores dos elementos em função do período de amostragem no Rio do Ouro, em Ouro Verde do Oeste-PR, entre novembro de 2012 e novembro de 2013.

\begin{tabular}{lcccccccccc}
\hline & $\mathbf{K}$ & $\mathbf{C a}$ & $\mathbf{M g}$ & $\mathbf{C u}$ & $\mathbf{Z n}$ & $\mathbf{M n}$ & $\mathbf{F e}$ & $\mathbf{C d}$ & $\mathbf{P b}$ & $\mathbf{C r}$ \\
\cline { 2 - 10 } & \multicolumn{7}{c}{$\mathbf{m g ~ L}^{-1}$} \\
\hline nov/12 & $1.28 \pm 0.14$ & $1.33 \pm 0.22$ & $1.35 \pm 0.11$ & $<0.005$ & $<0.005$ & $<0.01$ & $0.20 \pm 0.04$ & $<0.005$ & $0.06 \pm 0.13$ & $0.06 \pm 0.06$ \\
jan/13 & $1.48 \pm 0.24$ & $2.01 \pm 0.80$ & $1.36 \pm 0.12$ & $<0.005$ & $<0.005$ & $<0.01$ & $0.13 \pm 0.04$ & $<0.005$ & $0.04 \pm 0.10$ & $0.09 \pm 0.04$ \\
mar/13 & $1.69 \pm 0.68$ & $1.23 \pm 0.12$ & $1.34 \pm 0.14$ & $<0.005$ & $<0.005$ & $<0.01$ & $0.30 \pm 0.16$ & $<0.005$ & $0.01 \pm 0.01$ & $0.04 \pm 0.06$ \\
mai/13 & $1.01 \pm 0.36$ & $1.23 \pm 0.31$ & $1.28 \pm 0.20$ & $<0.005$ & $<0.005$ & $<0.01$ & $0.06 \pm 0.02$ & $<0.005$ & $0.05 \pm 0.08$ & $0.03 \pm 0.03$ \\
jun/13 & $1.22 \pm 0.44$ & $1.27 \pm 0.22$ & $1.24 \pm 0.19$ & $<0.005$ & $<0.005$ & $<0.01$ & $0.09 \pm 0.06$ & $<0.005$ & $0.02 \pm 0.03$ & $0.09 \pm 0.09$ \\
set/13 & $0.36 \pm 0.26$ & $1.79 \pm 0.84$ & $0.90 \pm 0.34$ & $<0.005$ & $<0.005$ & $<0.01$ & $<0.01$ & $<0.005$ & $<0.01$ & $<0.01$ \\
out/13 & $0.50 \pm 0.15$ & $1.46 \pm 0.29$ & $1.09 \pm 0.43$ & $<0.005$ & $<0.005$ & $<0.01$ & $<0.01$ & $<0.005$ & $<0.01$ & $<0.01$ \\
out/13 & $0.36 \pm 0.18$ & $1.47 \pm 0.63$ & $1.07 \pm 0.55$ & $<0.005$ & $<0.005$ & $<0.01$ & $<0.01$ & $<0.005$ & $<0.01$ & $<0.01$ \\
nov/13 & $0.59 \pm 0.14$ & $1.64 \pm 0.42$ & $1.89 \pm 0.02$ & $<0.005$ & $<0.005$ & $<0.01$ & $<0.01$ & $<0.005$ & $<0.01$ & $<0.01$ \\
\hline
\end{tabular}

Fonte: Elaboração dos autores, 2014.

Nota: $\mathrm{n}=5$. Limite de quantificação: $\mathrm{Cu}=0.005 \mathrm{mg} \mathrm{L}^{-1} ; \mathrm{Zn}=0.005 \mathrm{mg} \mathrm{L}^{-1} ; \mathrm{Mn}=0.01 \mathrm{mg} \mathrm{L}^{-1} ; \mathrm{Fe}=0.01 \mathrm{mg} \mathrm{L}^{\mathrm{L}}$ ${ }^{1} ; \mathrm{Cd}=0.005 \mathrm{mg} \mathrm{L}^{-1} ; \mathrm{Pb}=0.01 \mathrm{mg} \mathrm{L}^{-1} ; \mathrm{Cr}=0.01 \mathrm{mg} \mathrm{L}^{-1}$.

Não foram encontrados valores médios para os teores de $\mathrm{Cu}, \mathrm{Zn}, \mathrm{Mn}$ e $\mathrm{Cd}$ em nenhum dos pontos de amostragem (Tabela 03).

Quanto aos elementos $\mathrm{Fe}, \mathrm{Pb}$ e $\mathrm{Cr}$ (Tabela 03), não foi encontrada grande oscilação quanto aos seus teores entre os pontos de coleta, indicando que apesar da existência de diferentes atividades agroindustriais na região da bacia, esses pontos apresentam concentrações homogêneas desses metais.

O fato de os pontos de amostragem não apresentarem diferença é um indicativo de igualdade entre os pontos de amostragem, porém, este resultado não indica ausência ou baixo impacto ambiental.

Os teores médios referentes aos metais (Tabelas 02, 03 e 04, e Figuras 03 e 04) indicam baixo impacto ambiental oriundo de atividades agroindustriais e urbana sobre as características toxicológicas do rio, porém, cabe salientar que estas atividades poderão causar maiores impactos ao referido curso hídrico futuramente, caso o manejo conservacionista das áreas marginais seja abandonado.

Apesar das variações quanto à precipitação pluviométrica apresentada na Figura 02, observase na Tabela 04 que foi encontrada variação quanto aos teores de metais e nutrientes durante o período amostral, indicando que a variação pluviométrica foi suficiente para resultar em variações das concentrações dos mesmos.

\section{Conclusões}

As concentrações de metais nas águas do Rio do Ouro de maneira geral foram baixas e inferiores aos valores permitidos pela legislação, sendo que alguns parâmetros apresentaram resultados inferiores ao limite de quantificação (o que sugere que estes metais estejam presentes em concentrações extremamente baixas) e outros apresentaram valores médios próximos do limite estabelecido pela Resolução do CONAMA e pela Resolução da Anvisa.

Cabe salientar que, para uma efetiva avaliação dos teores dos metais pesados, é necessária a sistematização de um número maior de pontos de amostragem, visando um acompanhamento mais preciso e pontual das fontes de poluição. Além de ser imprescindível a continuação do monitoramento, tendo em vista que a presença de atividade agrícola é constante e muito ativa na região.

\section{Agradecimentos}

À Pontifícia Universidade Católica do Paraná (Campus Toledo) pelo espaço físico concedido; à Prefeitura Municipal de Ouro Verde do Oeste - PR pela parceria; e ao Laboratório de Química Ambiental e Instrumental da Universidade Estadual do Oeste do Paraná, Campus Marechal Cândido Rondon. 


\section{Referências Bibliográficas}

BRASIL, Agência Nacional de Águas. Disponibilidade e demandas de Recursos hídricos no Brasil. Acesso em: 22 mar. 2014. Disponível em: <http://arquivos.ana.gov.br/planejamento/ estudos/sprtew/2/2-ANA.swf>

Agência Nacional de Águas. Região Hidrográfica do Paraná. Acesso em: 22 mar. 2014. Disponível em: <http://www2.ana.gov.br/Paginas/portais/bacias/parana.aspx>

Agência Nacional de Vigilância Sanitária. Resolução de Diretoria Colegiada - RDC n. ${ }^{\circ} 274$ de 22 de setembro de 2005.

Conselho Nacional do Meio Ambiente. Resolução n. ${ }^{\circ} 357$ de 17 de março de 2005. Diário Oficial da União, Brasília. 2012.

, Lei n. ${ }^{\circ}$ 12.651, de 25 de maio de 2012. Diário Oficial da República Federativa do Brasil, DF,

INSTITUTO DE GEOGRAFIA E ESTATístICA (IBGE). IBGE Cidades. Paraná, Ouro Verde do Oeste, 2013. Acesso em: 22 mar. 2014. Disponível em: <http://www.cidades.ibge.gov.br/xtras/home.php>

PARANÁ. Secretaria de Estado do Meio Ambiente e Recursos Hídricos (SEMA). Bacias Hidrográficas do Paraná. Curitiba: SEMA, 2013.

PHILIPPI JR, A. Saneamento, Saúde e Ambiente: fundamentos para um desenvolvimento sustentável. Barueri, SP: Manole, 2005.

SCHONS, Daniele Cristina et al. Monitoramento da qualidade das águas do Rio do Ouro, em Ouro Verde do Oeste - PR: análises microbiológicas. In: Congresso Nacional de Meio Ambiente de Poços de Caldas, 11., 2014, Poços de Caldas. Anais... Poços de Caldas, 2014. Acesso em: 3 out. 2014. Disponível em: <http://meioambientepocos.com.br/portal/anais/2014/index.php>

WELZ, B.; SPERLING, M. Atomic absorption spectrometry. Weinheim: Wiley-VCH, 1999. 941 pp. 